\title{
Analysis of Phosphatase Activity from Aquatic Heterotrophic Bacteria at the Single Cell Level by Flow Cytometry: Example of a Development Achieved in the Regional Flow Cytometry Platform for Microbiology (Precym) Hosted by the Oceanology Center of Marseille
}

\author{
Gérald Grégori, Michel Denis, Solange Duhamel, France Van Wambeke, and Louins Mebarek
}

\begin{abstract}
Marine microbes play a very important role in biogeochemical cycles. Because of their biodiversity, the diversity of their metabolisms, and their physiological heterogeneity (live/dead, active/inactive cells), it is necessary to study them at the single cell level.

Flow cytometry has become a technique of choice to reach this goal, providing a fast, reliable, and multiparametric analysis of individual cells. However, the cost of such a technique makes flow cytometry out of reach for small institutes or laboratory units. It also requires a qualified and well-trained operator.

To make this technique available to all the microbiologists of the "Provence Alpes Côte d'Azur" Region, a Regional Flow Cytometry Platform (PRECYM), hosted by the Oceanology Centre of Marseille, was created by a consortium of six laboratories. Its goal is to provide researchers with the possibility to consider new approaches and to guarantee optimal technical, economic, and scientific exploitations of these instruments.

An example of such a technical development is described in this chapter. The ELF97 phosphatase substrate has been shown to be a performing tool to assess the phosphorus status of phytoplankton populations.

Recently, this technique has been successfully applied to marine heterotrophic bacteria in culture samples in which ELF-labeling was observed by microscopy.
\end{abstract}

G. Grégori $(\bowtie)$

Laboratoire de Microbiologie, Géochimie et Ecologie Marines, Université de la Méditerranée, Centre d'Océanologie de

Marseille, Campus de Luminy, UMR 6117, Case 901 13288,

Marseille, France

e-mail: gerald.gregori@univmed.fr

Lounis Mebarck

Docteur ( $\mathrm{Ph} \mathrm{D}$ ) en science de 1' environnement marin,

Observateur scientifique en mer, resposable de la planification,

e-mail: lounis.mabarek@yahoo.com
In this study, we describe a new protocol for ELF-labeling of heterotrophic bacteria that allow detection by flow cytometry. ELF-labeled samples were stored in liquid nitrogen for up to 4 months before analysis without any significant loss of total or ELFlabeled cell abundance and of relative ELF fluorescence intensity.

This method enables studying ectoenzymatic alkaline phosphatase activity of heterotrophic bacteria at the single cell level in environments even with low cell abundance. It has been successfully applied in both fresh and marine samples.

This approach is set to improve our understanding of the physiological response of heterotrophic bacteria to phosphorus limitation.

\section{Introduction}

The oceans cover more than $70 \%$ of the earth (Lalli and Parsons 1997). Most of the marine organisms are actually microscopic and unicellular. Aquatic microorganisms present a wide range of size from 0.2 to 100 $\mu \mathrm{m}$ and make up to $50 \%$ of the total biomass of the planet. They play crucial roles in the functioning of the Earth's biosphere and dominate the marine ecosystem in terms of biomass, with a high rate of turnover (Azam et al. 1983). They are responsible for:

(i) $\mathrm{CO}_{2}$ uptake for organic matter production (about half of our planet's annual primary production)

(ii) Most of the organic matter $(\mathrm{OM})$ mineralization in the oceanic water column $\left(\mathrm{CO}_{2}\right.$ release $)$

(iii) Playing an essential role in regulating the climate (contribution to the atmospheric $\mathrm{CO}_{2}$ sequestration 
in the deep ocean; producing chemically active biogases)

(iv) Ecosystem and public health concerns linked to the toxicity of certain species

Because of their biodiversity (including algae, protozoa, bacteria, archaea), their important biomass, and their large panel of metabolisms that make them ubiquist, they represent a key value in aquatic systems, and in particular in the cycle of chemical elements (carbon, nitrogen, phosphorus). To understand and apprehend the relationships between these microorganisms and their ecosystem, it is of prime importance to identify (at the species level), quantify (abundances), and characterize (cell activities) these microorganisms, which are often present in different physiological states (live, active, inactive, damaged, and dead). These studies imply analyses at the single cell level, and flow cytometry, together with fluorochromes, is the technique of choice to get fast and accurate data representative of the whole populations identified within a heterogeneous natural sample (Burkill 1987).

The need for such a technology led to the creation of the Regional Flow Cytometry Core Facility PRECYM (Plateforme Régionale de Cytométrie pour la Microbiologie) created by the synergy of several laboratories (for more details, see http://precym.com. univ-mrs.fr). The goal of this facility is to provide laboratories and scientists with the most advanced technology and protocols, and thus offer them the possibility to consider new approaches in their studies and opportunities in their work they could not afford without these instruments.

\section{Flow Cytometry Principle}

During the last decade, flow cytometry has become a very powerful technique and has impacted a wide range of fields from basic cell biology to genetics, immunology, molecular biology, microbiology, and environmental science. The principle of flow cytometry can easily be understood from its definition: measurement of the optical properties of cells (cyto-) flowing at the very center of a liquid sheath as they are individually intercepted by a light source focused at a very small volume. Particles (i.e., cells) are examined in a very short time $(2-10 \mu \mathrm{s})$, during which several optical signals are detected and collected, mainly light scatter and fluorescence emissions in the visible spectrum.

Signals coming from the cells are photons either from the laser and scattered by the cells, or produced by the cell fluorescence. Several optical filters are used to separate the different photons according to their wavelengths and drive them to the photodetectors (most often photodiodes for light scatter and photomultiplier tubes for fluorescences). Data are then displayed and interpreted to discriminate clusters of particles based on statistical analyses of the variables collected.

\section{Why Is Flow Cytometry So Popular Among Microbiologists?}

Flow cytometry analyses are very fast (up to several thousand cells per second). A very large number of cells can thus be analyzed per sample (up to 10 millions!), and the statistical results are obviously representative of the population. This also allows analyzing more samples, a key issue in spatial or temporal studies. Using these statistical analyses, it is possible to electronically separate these populations and identify them using multivariate analytical techniques. Flow cytometers can nowadays readily analyze single cells at rates up to 30,000 per second. Flow cytometry provides multiparametric analyses at the single cell level (several scatters and fluorescences). The data are quantitative data and can be correlated to other biochemical data. Moreover, data are acquired in real time.

The size class distribution of microorganisms can be assessed from the scattered light, and the analysis also provides the cell abundance. The presence of unique identification markers in the cells allows separating cell clusters. These markers can be natural in the case of auto-fluorescent cells (example: natural fluorescent pigments) or induced by adding fluorescent dyes (fluorochromes) that target particular cell components or activity.

The last, but not least, is that some flow cytometers, called "sorters," are able to physically separate the cells of interest. Upon sorting, cells can be collected in 
a tube, a Petri dish, a 96-well plate, etc. In most cases, sorting does not affect viability, and post-cultures are possible. Sorting is also very valuable for post-analyses (molecular biology).

Unfortunately, flow cytometers are very expensive instruments, very often out of reach for laboratories. Moreover, they require highly trained people. This is why the "Plateforme Régionale de Cytométrie pour la Microbiologie" PRECYM was created in 2005.

\section{The Regional Flow Cytometry Platform for Microbiology (PRECYM)}

Aware of the potential of this tool, the three laboratories of the COM (LMGEM UMR 6117, LOB UMR 6535, and DIMAR UMR 6540), as well as two Research Units of the IRD (UR 99 Cyroco and UR 103 CAMELIA), and the Laboratoire de Chimie Bactérienne (LCB UPR CNRS 9043) organized a consortium with the aim of acquiring a flow cytometer cell sorter (a MoFlo from DAKO, Dk). The Regional Flow Cytometry Platform PRECYM was created in 2005 and centralizes this equipment and know-how and makes them available to other units/institutes. PRECYM also allows the optimal technical, economic, and scientific exploitations of the instruments. PRECYM is also involved in the R\&D and in the technological survey, as well as the research valorization.

\subsection{Example of development achieved in PRECYM: Detection of phosphatase activity of heterotrophic prokaryotes characterized at the single cell level by flow cytometry}

The analysis of heterotrophic prokaryotes at the single cell level provides a very valuable insight in ecological studies. Archaea and bacteria, hereafter referred to as "bacteria" in the text, are indeed the major actors in the mineralization of the organic matter in the ocean. They are responsible for most of the aerobic respiration in the ocean. This respiration leads to the production of metabolic $\mathrm{CO}_{2}$, a greenhouse effect gas of special concern in the context of global warming. Most of the species (or strains) are still unknown, which makes bacteria the major source of unknown biodiversity. Moreover, bacteria can be present in different physiological states ranging from live and active, to live and inactive, and dead. When inorganic phosphorus (Pi) becomes a limiting element in the environment, some bacteria are able to break down exogenous organic phosphate compounds to utilizable inorganic forms (Pi) through alkaline phosphatase activity (APA). This phosphorus could then become available for the rest of the ecosystem. The fluorochrome ELF (Enzyme Labeled Fluorescence) from Molecular Probes (Eugene, Oregon, USA) targets APA at the single cell level. Such a method has been developed on phytoplankton, but studies on bacteria are scarce, and were made by microscopy.

ELF-P is a colorless substrate (i.e., not fluorescent). When ELF-P is hydrolyzed by alkaline phosphatases, it is transformed into a green fluorescent insoluble product (ELF alcohol ELF-A), which precipitates at the site of the enzymes, inducing a bright green fluorescence within the cells when they are excited by a UV light. We have thus developed a protocol to detect phosphatase activity of heterotrophic bacteria characterized at the single cell level by flow cytometry. The method was first developed on bacteria in cultures (Duhamel et al. 2008b). It was then applied to natural fresh and marine samples (Duhamel et al. 2008a, b). By simultaneously incubating the cells with a fluorescent nucleic acid dye (such as DAPI or $\mathrm{PI}$ ), it is then possible to detect all the bacteria, and the fraction responsible for the phosphatase activity (Fig. 1).

The protocol has been specially designed for freshwater and marine natural samples. It takes into account the need for cell concentration (when the abundance is too low) and the disaggregation step mandatory to get individual bacteria. This protocol is complementary to what is observed by microscopy. Microscopy analyses are qualitative (a cell expresses or not the fluorescence). To be quantitative (how much does a cell express the activity), microscopy must be coupled with advanced image analysis, and it then becomes highly time-consuming. Flow cytometry is instead quantitative and much faster. 


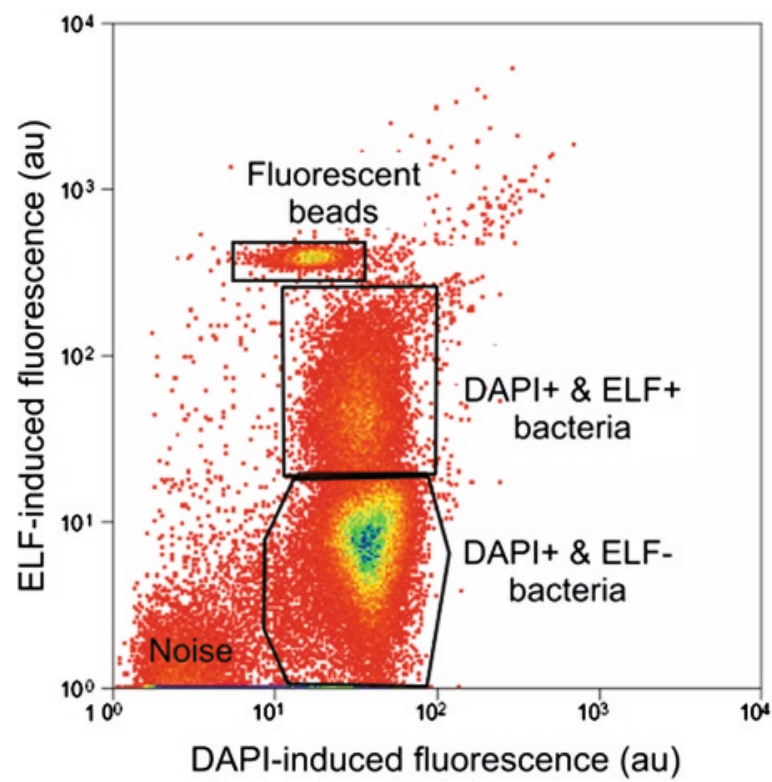

Fig. 1 A double staining by ELF and DAPI makes it possible to discriminate by flow cytometry between bacteria expressing (DAPI positive and ELF positive) or not (DAPI positive and ELF negative) alkaline phosphatase activity. DAPI is a blue fluorescent nucleic acid dye that is used to stain all the bacteria and discriminate them from the debris (noise). ELF is a substrate, which is fixed and transformed by the alkaline phosphatase into a bright green fluorescent crystal. Each dot on the figure represents a single particle (i.e., a bacterium). The color codes for the abundance. Fluorescent beads $(2 \mu \mathrm{m}$ diameter $)$ are added in the sample as internal control to monitor the instrument during the analysis and standardize the intensities between samples

\section{Conclusion}

The goal of this work is to apply this approach in various natural conditions. Combining the detection and quantification of APA at the single cell level for both phytoplankton and bacteria will bring new insights to the comprehension of algal-bacterial competition for bioavailable organic phosphorus when inorganic phosphorus is a limiting factor. Combining this ELF labeling protocol with molecular techniques such as fluorescent in situ hybridization (FISH) will be very valuable for identifying the phylogenic groups responsible for APA.

\section{References}

Azam F, Fenchel T, Gray JG, Meyer-Reil LA, Thingstad TF (1983) The ecological role of water-column microbes in the sea. Marine Ecology Progress Series 10:257-263

Burkill PH (1987) Analytical flow cytometry and its application to marine microbial ecology. In: Sleigh MA (ed) Microbes in the sea. Ellis Horwood Ltd., Chichester, pp 139-166

Duhamel S, Gregori G, Mauriac R, Van Wambeke F, Nedoma J (2008a) Detection of extracellular phosphatase activity at the single cell level by flow cytometry. Cytometry 75:163-168

Duhamel S, Gregori G, Van Wambeke F, Mauriac R, Nedoma J (2008b) A method for analysing phosphatase activity in aquatic bacteria at the single cell level using flow cytometry. J Microbiol Meth 75:269-278

Lalli CM, Parsons TR (1997) Biological oceanography, an introduction, 2nd edn. Elsevier, Amsterdam, 313 pp 